Cytologia Focus:

\title{
Chromosome Microdissection and Microcloning: Technique and Application in the Plant Sciences
}

\author{
Maoyin Sheng ${ }^{1,2,3 *}$, Mengdi Gao ${ }^{2,3}$, Linjiao Wang ${ }^{2,3}$ and Xuelian Ren ${ }^{1}$ \\ ${ }^{1}$ Molecular Genetics Key Laboratory of China Tobacco, Guizhou Academy of Tobacco Science, \\ Guiyang, 550081, China \\ ${ }^{2}$ National Engineering Research Centre for Karst Rocky Desertification Control, Guizhou Normal University, \\ Guiyang, China \\ ${ }^{3}$ Guizhou Engineering Laboratory for Karst Rocky Desertification Control and Derivative Industry, Guiyang, China
}

Received January 30, 2020; accepted March 5, 2020

\begin{abstract}
Summary The technique of chromosome microdissection and microcloning, as a bridge between cytogenetics and molecular genetics, has been widely applied and play an important role in many genetic researches, such as chromosome painting probe isolation, genetic linkage map and physical map construction, and expressed sequence tags generation. To promote the further application of this technique in plants, in the present study, firstly, the experimental procedure was summarized. Secondly, the application status of this technique in plant researches was reviewed and the intractable problems of the application in plant researches were elucidated. Finally, the application of this technique in plants was looked into in the future. The results of the present study have obvious significance in promoting the application of chromosome microdissection and microcloning technique in plants.
\end{abstract}

Keywords Chromosome, Microdissection, Microcloning, Technique, Application.

Chromosome microdissection and microcloning, as a bridge between cytogenetics and molecular genetics, has been widely applied and play an important role in many studies, such as chromosome painting probe isolation, genetic linkage map, and physical map construction, and expressed sequence tags generation. Chromosome microdissection and microcloning, a technique in which the target chromosome/segment is cut or burned by glass microneedles or lasers in an inverted microscope for separation from other chromosomes and the isolated chromosome/segment is then collected and amplified in vitro to establish specific DNA libraries (Emmert-buck et al. 1996). The specific DNA libraries are mainly established by the direct cloning of the enzyme and the PCR-mediated microcloning technique. This technique was developed and first used to cut the chromosomes of Drosophila melanogaster (Scalenghe et al. 1981). After the creation of the DNA PCR technique, chromosome microdissection and microcloning developed rapidly. However, it is still mainly applied to studies of human and animal chromosomes (Zadesenets et al. 2017, Mahalingam 2018). The application of chromosome microdissection in plant research is limited because (1) the thick cell wall, denser cell cytoplasm, and asynchronous cell division make chromosome preparation difficult in plants and (2) chromosome banding is not perfect in

\footnotetext{
* Corresponding author, e-mail: shmoy@163.com

DOI: $10.1508 /$ cytologia.85.93
}

most plants, so it is very difficult to distinguish the target chromosome (Zadesenets et al. 2017). Nevertheless, the technique of chromosome microdissection and microcloning was successfully used in rye chromosomes by Saudery et al. (1991), and the cloning of rye DNA was successfully achieved. Since then, the application of this technique in the field of plant research has rapidly developed (Casson et al. 2005, Deng et al. 2014, Cao et al. 2017).

The advantage of chromosome microdissection is that any chromosome or DNA marker can be accurately obtained for any chromosome region according to the research need (Burgemeister 2005). Although the research and application of the chromosome microdissection technique in plants had a late start, with the rapid and strong development of plant genome research, chromosome microdissection will indeed become a powerful tool for the localization and cloning of plant genes (Day et al. 2005). It will also play a unique role in elucidating the structure of the plant genome, and studies on plant functional genomics will generate a new research field in plant cytogenetics. In this article, the experimental procedure of this technique was summarized. Then, the application of this technique in the field of plant research was reviewed. Finally, the key scientific problems and research prospects of this technique in plant research were discussed. The purpose of this article is to provide references for further studies and applications of this technique in the field of plant research. 


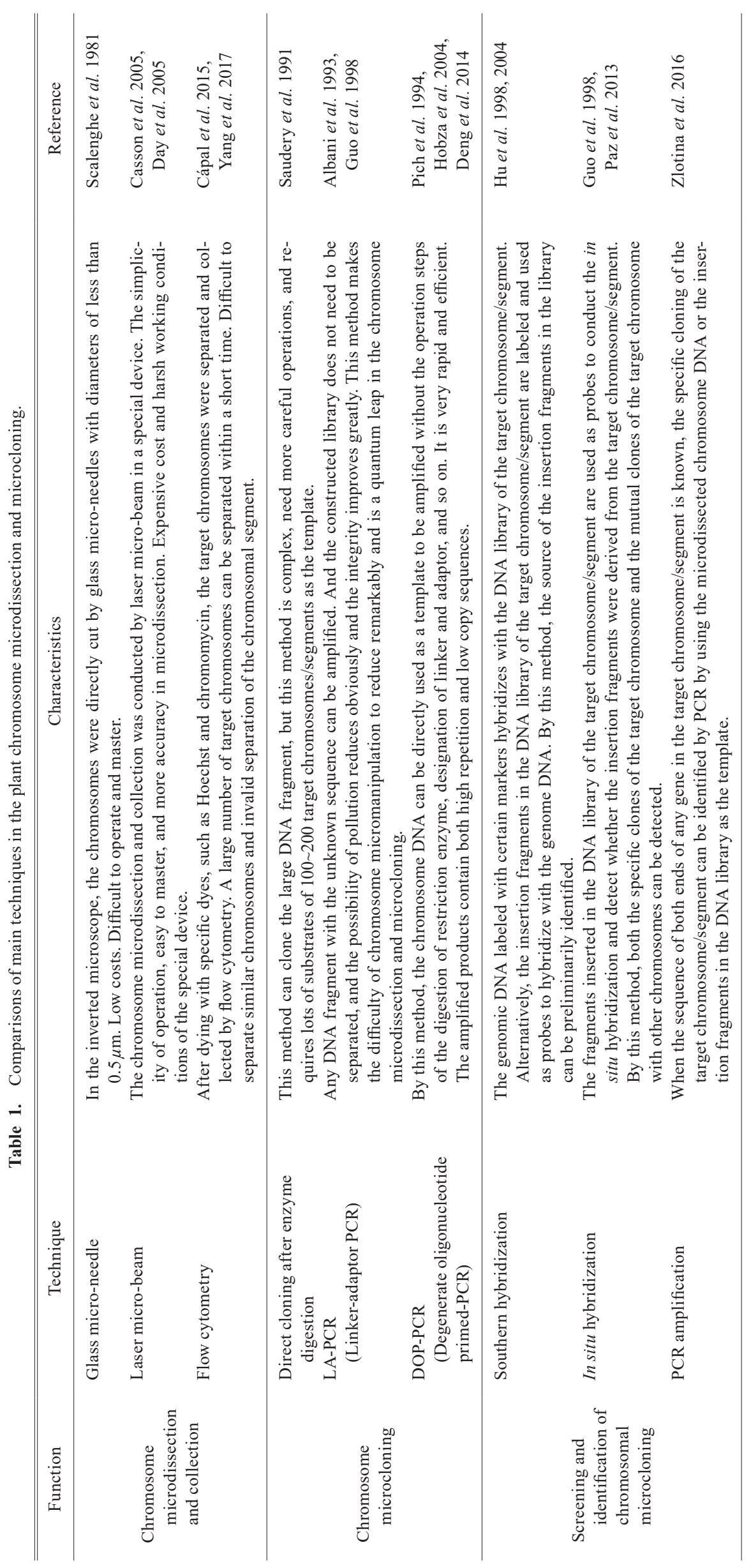


Table 2. The commercial products for chromosome laser microdissection.

\begin{tabular}{|c|c|c|c|c|c|}
\hline No. & Company & Country & Product & Principle & Comparison \\
\hline 1 & $\begin{array}{l}\text { Arcturus/Life } \\
\text { Technologies }\end{array}$ & USA & Arcturus XT & $\begin{array}{l}\text { A thermoplastic membrane of ethylene-vinyl } \\
\text { acetate (EVA) was heated by a low-energy } \\
\text { infrared laser pulse, and the targets adhered in } \\
\text { the membrane under a microscope. }\end{array}$ & \multirow{4}{*}{$\begin{array}{l}\text { The product of Arcturus XT } \\
\text { is a unique microdissection } \\
\text { instrument combining la- } \\
\text { ser capture microdissection } \\
\text { (LCM) and ultraviolet (UV) } \\
\text { laser cutting. The products } \\
\text { of the other three companies } \\
\text { all use ultraviolet lasers to } \\
\text { accomplish microdissection } \\
\text { and target collection. }\end{array}$} \\
\hline 2 & Leica & Germany & $\begin{array}{l}\text { LMD6500, } \\
\text { LMD7000 }\end{array}$ & $\begin{array}{l}\text { The targets were microdissected by an ultra- } \\
\text { violet laser micro-beam through a microscope } \\
\text { lens and collected by gravity. }\end{array}$ & \\
\hline 3 & Zeiss & Germany & $\begin{array}{l}\text { PALM } \\
\quad \text { MicroBeam }\end{array}$ & $\begin{array}{l}\text { The targets were microdissected by a focused } \\
\text { laser micro-beam and bounced into tubes for } \\
\text { collection by the pressure wave of the laser. }\end{array}$ & \\
\hline 4 & $\begin{array}{l}\text { MMI (Molecular } \\
\text { Machine Industry) }\end{array}$ & Switzerland & $\begin{array}{r}\text { Cell Ector Plus, } \\
\text { Cell Cut Plus }\end{array}$ & $\begin{array}{l}\text { The targets were microdissected and collected } \\
\text { by the solid-state ultraviolet laser. During the } \\
\text { microdissection process, the laser does not } \\
\text { contact the targets and only slightly damages } \\
\text { the targets. }\end{array}$ & \\
\hline
\end{tabular}

\section{Experimental procedures}

The chromosome microdissection and microcloning technique can be divided into five steps: 1) preparation of the chromosome specimen, 2) identification of target chromosomes, 3) microdissection and collection of target chromosomes or chromosomal segments, 4) construction of the microcloning library and 5) screening and identification of microclones.

\section{Identification of target chromosomes}

The chromosome specimens must be prepared precisely by conventional methods. After the preparation of chromosome specimens, the appropriate methods must be used to identify the target chromosomes. Karyotype analysis and chromosome banding are often used. For example, the meiotic monovalent chromosomes in wheat monosomic lines were identified by karyotype analysis and successfully microdissected (Vega et al. 1994). The B chromosomes of rye were identified by chromosome banding and successfully microdissected (Houben et al. 2001). However, traditional methods of karyotype analysis and chromosome banding are limited in the identification of chromosomes for microdissection, especially in these species with small and inconspicuous chromosomes.

\section{Construction of microcloning library}

The microdissection and collection of target chromosomes/segments can be conducted by the techniques of glass micro-needles, laser micro-beams, and flow cytometry (Table 1). Currently, there are special laser beam devices (Table 2) for the chromosome microdissection. After microdissection, the obtained chromosomes/segments can be amplified in vitro for subsequent studies. The construction of chromosome microcloning libraries has mainly been conducted by two methods: direct cloning after enzyme digestion and PCR-mediated microcloning (Table 1).

Direct cloning after enzyme digestion-Direct clon- ing after enzyme digestion was widely applied before the construction of PCR. The procedure for this method includes the following steps. First, the target chromosomes/segments were extracted from DNA. Then, the extracted DNA was digested by enzymes after deproteinization and purification. Third, the DNA digested was added to the ligase to link the vector digested by the same restriction enzyme. Finally, the DNA linked to the vector was transformed into the host bacteria to construct the chromosome DNA library. Saudery et al. (1991) were the first to apply this method in plant chromosome microcloning and DNA library construction. They cut and separated the B chromosomes of rye by glass micro-needles and successfully microcloned the B chromosomes after extraction, deproteinization, purification, digestion, and so on (Saudery et al. 1991).

PCR-mediated microcloning- The number of target chromosomes obtained directly by microdissection is limited. After PCR amplification in vitro, additional target chromosomes can be obtained to meet the needs of molecular biology experiments. Since 1989, several PCR-mediated microcloning methods have been developed (Deng et al. 2014, Cápal et al. 2015). Two of these methods, LA-PCR and DOP-PCR, are used frequently.

LA-PCR (Linker adaptor PCR) involves the following steps. First, the DNA of the target chromosomes/segments was digested by restriction enzymes. Second, according to the base sequence of the sticky end produced after enzyme digestion, one linker and one adaptor were designed, and the mixture of the linker and adaptor can produce the same sticky ends as those of the DNA digested by restriction enzymes. Finally, the PCR amplification was conducted by using the linker as the primer. Albani et al. (1993) successfully applied the linkeradaptor system of Sau3AI for the PCR amplification of microdissected chromosomes. Guo et al. (1998) have also successfully cloned the microdissected B chromosome of rye by LA-PCR with the linker-adaptor system of Sau3AI. 
DOP-PCR (Degenerate oligonucleotide-primed PCR) refers to the use of a simple degenerate primer for amplification after two PCR reactions (Pich et al. 1994). The annealing temperature is low in the first PCR reaction. The chromosomes of bean and wheat were microdissected and successfully amplified by DOP-PCR (Pich et al. 1994, Deng et al. 2014).

\section{Screening and identification of microclones}

The specificity of the constructed DNA library is generally screened and identified by using Southern hybridization, in situ hybridization and the PCR amplification of specific DNA segments (Table 1) (Paz et al. 2013).

Southern hybridization can preliminarily identify the source of the inserted DNA fragments in the library. Hu et al. (1998) successfully proved that the microclones produced from in vitro PCR were derived from the maize genome by Southern hybridization of digoxinlabeled maize genomic DNA with single chromosome DNA microdissected from maize. Using the same method, the 4 DNA fragments in the library of the $6 \mathrm{~B}$ wheat chromosome were proved to be derived from the wheat genome ( $\mathrm{Hu}$ et al. 2004). In situ hybridization not only detects the chromosomal source of the inserted DNA fragments in the library and identifies the specific clones of the target chromosome, but it also identifies the mutual clones of the target chromosome with other chromosomes (Paz et al. 2013). By in situ hybridization, Guo et al. (1998) successfully located the PCR products at the terminal of the $\mathrm{B}$ chromosome of rye. When the sequence of both ends of any gene in the target chromosome/segment is known, the specific cloning of the chromosome can be identified by PCR. The 4 different DNA fragments of R1, R2, R3 and R4 were successfully proved to be derived from the wheat $6 \mathrm{~B}$ chromosome by PCR using different SSR markers of the $6 \mathrm{~B}$ chromosome as primers (Hu et al. 2004).

\section{Applications in plant studies}

Construction of a specific DNA library for a single chromosome/segment

After chromosome microdissection, the length of the DNA fragments obtained by PCR amplification is generally $\sim 150-2000 \mathrm{bp}$, which is suitable for constructing a specific DNA library (Liu et al. 2004, Hwang and Lim 2011, Shibata et al. 2017). Until recently, the DNA library for a single whole chromosome has been constructed in oat (Emmert-buck et al. 1996), corn (Hu et al. 1998), and lily (Hwang and Lim 2011). A DNA library for chromosomal segments has been constructed by the same method, such as the short arm of $1 \mathrm{H}$ chromosome of barley (Schondelmaier et al. 1993) and the long arm of the $6 \mathrm{~B}$ chromosome of common wheat $(\mathrm{Hu}$ et al. 2004).
Construction of a specific probe pool for a single chromosome/segment

The product obtained from the PCR of the microdissected chromosome/segment is the probe pool. The probe pool combined with fluorescence in situ hybridization (FISH) is a powerful technique for studying the chromosome structure and its variations, such as chromosome translocation, inversion (Taguchi et al. 2016, Yanagi et al. 2017). Deng et al. (1999) successfully set up a specific probe pool for the fourth pair of chromosomes of rice, and the specificity was identified by FISH. This research laid the foundation for screening the new specific molecular markers from the DNA library of a single chromosome in rice.

Development of new molecular markers tightly linked to the target genes

Molecular marker-assisted breeding must have a sufficient number of molecular markers that are tightly linked to the target genes. However, effective molecular markers that can be utilized for breeding are currently lacking, obviously limiting the further development and application of molecular marker-assisted breeding (Doležel et al. 2014). Thus, it is important and significant to develop new molecular markers for breeding. The length of the microdissected DNA fragment is typically $1-3 \mathrm{Mb}$ when using the chromosome microdissection technique. Thus, screening molecular markers from the DNA library for the target chromosome/segment constructed by chromosome microdissection and microcloning will become an extremely effective method for developing new molecular markers tightly linked with the target genes (Cao et al. 2017). Moreover, from the specific DNA library, the efficiency of developing new molecular markers can be greatly improved (Seifertova et al. 2013). Based on the microdissection of the short arm of barley $1 \mathrm{H}$ chromosome, the genetic linkage map of molecular markers for the chromosomal segment was constructed after microcloning, and one RFLP marker tightly linked to the gene $(M a)$ for resistance to powdery mildew was screened (Schondelmaier et al. 1993). The long arm of the barley $3 \mathrm{H}$ chromosome was microdissected and collected, and one moderately repetitive sequence and one highly repetitive sequence were obtained after microcloning (Bush et al. 1995). The results provided a foundation for the development of microsatellite markers in barley.

Construction of a dense linkage map matching the physical map

The efficiency of developing new molecular markers can be greatly improved by microdissection and microcloning, which is extremely significant for the construction of a dense and homogeneous genetic linkage map. To date, molecular linkage maps have been constructed for many plant species, such as rice, soybean, corn, and 
tobacco, but except for a few species of rice, the genetic linkage map of most plants is highly unsaturated, and the distribution of genetic markers in the linkage group is not homogeneous (Doležel et al. 2014). The development of new molecular markers from the DNA library of the target chromosome segment by chromosome microdissection and microcloning can increase the density of genetic linkage maps and resolve the problem of the nonhomogeneous distribution of molecular markers.

Also, there is an important problem, that is, the genetic linkage map does not match its physical map, whether in the traditional genetic linkage map or the molecular marker linkage map. Occasionally, the distance of genes showing in the genetic linkage map greatly differs from the physical distance in the chromosome. Routinely, the relationship of the genetic linkage map with its chromosomal physical map was conducted by chromosome terminal deletion (Doležel et al. 2014). However, the breeding of the chromosome terminal deletant takes several years, and the number of chromosome terminal deletants that can breed is limited. Thus, new techniques to resolve the relations of the genetic linkage map with its chromosomal physical map are urgently needed. By chromosome microdissection and microcloning, specific molecular markers of the target chromosome segment can be obtained, which can help quickly realize the relationship between the genetic linkage map and its physical map. In addition, the single-copy molecular markers of STS, obtained from the specific DNA library of the target chromosome/segment constructed by chromosome microdissection and microcloning, can be used to construct both of the genetic linkage map and the physical map. And the corresponding relationship between the genetic map and physical map can be achieved by this method.

\section{Localization and cloning of genes}

Specific probes obtained by microcloning is an effective method to clone new genes (Roux et al. 2014, Cao et al. 2017). The $1 \mathrm{D}$ chromosome of wheat was microdissected and separated in 2009, and the gene fragments for the subunit (1Dx5) of hmw-glutenin (HMW-GS) were cloned after the microcloning (Tian et al. 1999). Moreover, the microdissection and microcloning of a specific chromosome carrying target resistant genes can be used for gene cloning to achieve target resistance. Wan et al. (2000) used glass needles to microdissect and separate pairs of chromosomes carrying the BYDV (barley yellow dwarf virus) resistance gene in the wheatThinopyrum alien additional line TAI-27. Then, based on the successful microdissection of a chromosome carrying anti-BYDV genes in the wheat-Thinopyrum alien additional line TAI-27, the cloning of the resistance genes was effectively conducted (Jiang et al. 2004).
Applications in plant evolutionary genetics

The specific DNA library for the target chromosome/ segment, constructed by chromosome microdissection and microcloning, can be used as probes to conduct the micro-FISH (Zlotina et al. 2016, Han et al. 2017, Kosyakova et al. 2017). By this method, the differentiation and homology of the target chromosome/segment between species can be clarified and their evolutionary relationship can be established, thus providing a new way to study plant evolutionary genetics and comparative genomics. Zhang et al. (2006) constructed a probe pool of the centric region of the rye B chromosome by chromosome microdissection and microcloning, and by FISH, the highly homologous DNA of the centric region between rye A and B chromosomes was identified. Houben et al. (2001) studied the origin and evolution of the rye $\mathrm{B}$ chromosome based on the DNA library construction of $\mathrm{B}$ chromosome/segment by chromosome microdissection and microcloning. The results showed that the $\mathrm{B}$ chromosome was derived from the A chromosome but lacked the repetitive sequence family contained in the heterochromatin region of the A chromosome (Houben et al. 2001).

\section{Intractable application to plant research}

Difficult preparation of high-class chromosome specimens

The preparation of chromosome specimens is the most fundamental and key technology affecting chromosome microdissection. However, the solid cell wall and nonsynchronous cell division often result in the unsatisfactory dispersion of chromosomes with an unclean background in the preparation of chromosome specimen, seriously affecting the effect of chromosome microdissection. Another major concern for the successful application of chromosome microdissection and microcloning was the extensive depurination of the chromosomal DNA caused by acid treatment during sample fixation in the chromosome preparation. Currently, depurination can be reduced or avoided by improving the chromosome preparation procedure.

Obvious difficulties in the identification of target chromosomes

The ploidy of the plant genome is multiform, the variation of chromosome structure is remarkable, and the gene sequence is also not highly conservative (Doležel et al. 2014). Thus, in plants with small similar chromosomes, it is impossible to accurately identify the target chromosomes and their fine structures. Although the technique of chromosome banding is helpful for the identification of target chromosomes, this technique can destroy the DNA structure because of plant chromosomes with a high ratio of DNA and a low ratio of protein (Doležel et al. 2014), detrimentally constructing the complete DNA library for the target chromosome. 
Thus, the application of chromosome banding for chromosomal identification in chromosome microdissection and microcloning is limited.

The huge workload of screening the chromosome DNA library

The DNA content of plant chromosomes with a large number of highly repetitive sequences is high (Seifertova et al. 2013). For example, the genome content ( $C$ value) of wheat is $1.6 \times 10^{11} \mathrm{bp}$, and the average of each chromosome is $0.8 \times 10^{10} \mathrm{bp}$, with approximately $80 \%$ repetitive sequences (Hu et al. 2004). If the length of each insert fragment is $1 \mathrm{~kb}$, then the specific DNA library for each chromosome will contain more than $8 \times 10^{7}$ clones. In addition, wheat is an allohexaploid with the chromosome complement of AABBDD $(2 n=6 x=42)$. Thus, the specific DNA library for all 42 chromosomes of the wheat genome will contain $3.36 \times 10^{9}$ clones, and screening the chromosome DNA library will be an immense task.

Unavoidable contamination of cytoplasmic DNA

PCR-mediated cloning of microdissected chromosome DNA made chromosome microdissection and microcloning more practicable. However, it is difficult to completely avoid DNA contamination from the cytoplasm, foreign species, and non-target chromosomes. The amplification of non-target DNA will greatly affect the quality of chromosome-specific DNA libraries. Generally, in plant chromosome microdissection and microcloning, DNA contamination can come from the following three sources: (1) foreign species, (2) nontarget chromosomes, and (3) cytoplasmic DNA (Zhou and $\mathrm{Hu}$ 2007). DNA contamination by foreign species can be controlled by strict disinfection and sterilization of instruments and chemical reagents. DNA contamination by non-target chromosomes can also be avoided by careful operation during microdissection. However, the contamination of cytoplasmic DNA is a serious concern. Due to the particularity of plant cells, it is unavoidable that the slides of plant chromosome specimens will contain remnants from the cytoplasm and cell wall. Furthermore, the contamination of cytoplasmic DNA is almost unavoidable in chromosome microdissection and microcloning (Zhou and $\mathrm{Hu}$ 2007). Hu et al. (1998) proposed a method of adding a drop of $50 \%$ ethanol to the target chromosome to microdissect, thus reducing the contamination by cytoplasmic DNA. Also, using a single chromosome/segment as a template to conduct the PCR amplification is an effective means to reduce the contamination of cytoplasmic DNA (Yang et al. 2017).

\section{Plant chromosome microdissection and microcloning} technique: the future

The chromosome microdissection and microcloning technique has been widely used in the field of plant mo- lecular genetics (Zhou and Hu 2007). The location and cloning of genes and the construction of genetic linkage and physical maps are based on the operations of chromosomes. Thus, the chromosome microdissection and microcloning technique developed and combined with other advanced techniques in molecular biology, such as PCR, molecular marker development, and chromosomal painting, will have further and broader applications in plant research fields, such as gene location and cloning, specific probe development, constructing genetic linkage maps, and chromosomal evolution.

Although whole-genome sequencing has rapidly developed in plants since the start of the genome project, a serious problem in plant genome sequencing remains (Doležel et al. 2014, Zhang et al. 2006). The huge and polyploid genomes with high heterozygosity contain highly repetitive sequences and repeated fragments of whole/parts of genes, and accurate assembly after sequencing became an impossible task in plants. However, the sequence of a single chromosome can easily solve this intractable problem (Deng et al. 2018). Therefore, the chromosome microdissection technique can produce a single chromosome and will play an important role in the whole genome sequencing of plants in the future.

Although the flow cytometry to separate single chromosome are fast and accurate, the difficult and invalid to separate similar chromosomes limit the further application in genetics. Along with the improvement of FISH technique, especially, the development of specific DNA probes labeled fluorescent dyes and foundation of phase metaphase chromosome hybridization technique in liquids, the flow cytometry combined with FISH has been successfully applied in the separation of similar chromosomes. Kosyakova et al. (2017) successfully separated the similar chromosomes of humans by the flow cytometry combined with FISH technique. So it can be expected that the flow cytometry with FISH technique will be widely applied in the separation and collection of similar chromosomes in plants.

Although there are still several problems in the application of chromosome microdissection and microcloning in plants, with the continuous improvement of chromosome preparation, the development of enzyme digestion and PCR technique, the integration of glass micro-needles and laser microbeams, and the production and application of more sophisticated machines, the automatization of chromosome microdissection will be greatly improved, the microdissection will be more accurate, and the efficiency of microdissection and microcloning will increase markedly. Thus, we predict that chromosome microdissection and microcloning will play an important role in the whole genome sequencing, evolutionary genetics, molecular marker-assisted breeding, development of chromosome-specific probes, and gene location and cloning of plants. 


\section{Acknowledgements}

This work was financially funded by the Project Supported by the Open Foundation of Molecular Genetics Key Laboratory of China Tobacco (Guizhou Academy of Tobacco Science), the Support Plan for Excellent Young Science and Technology talents of Guizhou Province (Qiankehe Pingtai Rencai [2017]5638), and the Support Plan for Science and Technology Top-notch Talents of Guizhou Higher Education Institutions (Qianjiaohe KY zi [2016] 064).

\section{References}

Albani, D., Cote, M. J., Armstrong, K. C., Chen, Q., Segal, A. and Robert, L. S. 1993. PCR amplification of microdissected wheat chromosome arms in a simple single tube reaction. Plant J. 4: 899-903.

Burgemeister, R. 2005. New aspects of laser microdissection in research and routine. J. Histochem. Cytochem. 53: 409-412.

Bush, W., Martin, R., Herrmann, R. G. and Hohmann, U. 1995. Repeated DNA sequences isolated by microdissection. I. Karyotyping of barley (Hordeum vulgare L.). Genome 38: 1082-1090.

Cao, X., Liu, Y., Liu, Z., Liu, F., Wu, Y., Zhou, Z., Cai, X., Wang, X., Zang, Z., Wang, Y., Luo, Z., Peng, R. and Wang, K. 2017. Microdissection of the Ah01 chromosome in upland cotton and microcloning of resistance gene anologs from the single chromosome. Hereditas 154: 13.

Cápal, P., Blavet, N., Vrána, J., Kubaláková, M. and Dolezel, J. 2015. Multiple displacement amplification of the DNA from single flow-sorted plant chromosome. Plant J. 84: 838-844.

Casson, S., Spencer, M., Walker, K. and Lindsey, K. 2005. Laser capture microdissection for the analysis of gene expression during embryogenesis of Arabidopsis. Plant J. 42: 111-123.

Day, R. C., Grossniklaus, U. and Macknight, R. C. 2005. Be more specific! Laser-assisted microdissection of plant cells. Trends Plant Sci. 10: 397-406.

Deng, C., Bai, L., Li, S., Zhang, Y., Li, X., Chen, Y., Wang, R. R., Han, F. and Hu, Z. 2014. DOP-PCR based painting of rye chromosomes in a wheat background. Genome 57: 473-479.

Deng, K., He, F. and Shen, D. 1999. Microdissection and amplification of rice chromosome No. 4. J. Fuda Univ. 38: 444-450.

Deng, L., Zhang, M., Liu, T., Cao, W., Guo, J., Mao, C., Li, Y., Wang, J., Huang, W., Lu, D., Zhang, S., Li, Z. and He, J. 2018. Long range haplotyping of paired-homologous chromosomes by single-chromosome sequencing of a single cell. Sci. Rep. 8: 1640.

Doležel, J., Vrána, J., Cápal, P., Kubaláková, M., Burešová, V. and Šimková, H. 2014. Advances in plant chromosome genomics. Biotechnol. Adv. 32: 122-136.

Emmert-buck, M. R., Bonner, R. F., Smith, P. D., Chuaqui, R. F., Zhuang, Z., Goldstein, S. R., Weiss, R. A. and Liotta, L. A. 1996. Laser capture microdissection. Science 274: 998-1001.

Guo, G., Chen, C., Li, X., Song, W. and Chen, R. 1998. Cloning of the telomere associated sequence of rye B chromosomes. Acta Bot. Sin. 40: 1123-1128.

Han, H., Liu, W., Lu, Y., Zhang, J., Yang, X., Li, X., Hu, Z. and Li, L. 2017. Isolation and application of $P$ genome-specific DNA sequences of Agropyron Gaertn. in Triticeae. Planta 245: 425-437.

Hobza, R., Lengerova, M., Cernohorska, H., Rubes, J. and Vyskot, B. 2004. FAST-FISH with laser beam microdissected DOP-PCR probe distinguishes the sex chromosomes of Silene latifolia. Chromosome Res. 12: 245-250.

Houben, A., Field, B. L. and Saunders, V. A. 2001. Microdissection and chromosome painting of plant B chromosomes. Methods
Cell Sci. 23: 115-124.

Hu, Z., Dang, B., Zhou, Y., Cui, L., Wang, L., Zhang, T., Li, L. and Chen, Z. 1998. Isolation of single chromosome and DNA amplification in vitro in Zea mays. Acta Genet. Sin. 25: 545-550.

Hu, Z., Wang, H., Shi, R., Dang, B., Hu, J., Yin, W., Chen, Y., Jiang, S. and Zheng, H. 2004. Microdissection and construction of region-specific DNA libraries of wheat chromosome 6B. Acta Bot. Sin. 46: 1357-1365.

Hwang, Y. and Lim, K. 2011. Development of microdissection and chromosome specific genomic library in Lilium tigrinum. Genes Genom. 33: 451-455.

Jiang, S., Zhang, L., Hu, J., Shi, R., Zhou, G., Chen, Y., Yin, W., Wang, R. and Hu, Z. 2004. Screening and analysis of differentially expressed genes from an alien addition line of wheat Thynopyrum intermedium induced by barley yellow dwarf virus infection. Genome 47: 1114-1121.

Kosyakova, N., Liehr, T. and Al-rikabi, A. B. H. 2017. FISHMicrodissection. In: Liehr, T. (ed.). Fluorescence In Situ Hybridization (FISH). Springer Verlag, Berlin. pp. 235-254.

Liu, X., Wang, H., Li, Y., Tang, Y., Liu, Y., Hu, X., Jia, P., Ying, K., Feng, Q., Guan, J., Jin, C., Zhang, L., Lou, L., Zhou, Z. and Han, B. 2004. Preparation of single rice chromosome for construction of a DNA library using a laser microbeam trap. J. Biotechnol. 109: 217-226.

Mahalingam, M. 2018. Laser Capture Microdissection: Insights into Methods and Applications. In: Murray, G. (ed.). Laser Capture Microdissection: Methods in Molecular Biology. Humana Press, New York. pp. 1723: 1-17.

Paz, N., Zabala, A., Royo, F., García-Orad, Á., Zugaza, J. L. and Parada, L. A. 2013. Combined fluorescent-chromogenic in situ hybridization for identification and laser microdissection of interphase chromosomes. PLoS One 8: e60238.

Pich, U., Houben, A., Fuchs, J., Meister, A. and Schubert, I. 1994. Utility of DNA amplified by degenerate oligonucleotide-primed PCR (DOP-PCR) from the total genome and defined chromosomal regions of field bean. Mol. Genet. Genomics 243: 173-177.

Roux, B., Rodde, N., Jardinaud, M. F., Timmers, T., Sauviac, L., Cottret, L., Carrère, S., Sallet, E., Courcelle, E., Moreau, S., Debellé, F., Capela, D., Carvalho-Niebel, F. D., Gouzy, J., Bruand, C. and Gamas, P. 2014. An integrated analysis of plant and bacterial gene expression in symbiotic root nodules using lasercapture microdissection coupled to RNA sequencing. Plant J. 77: $817-837$.

Saudery, M. J., Forster, J. W., Macadam, S. R., Blunden, R., Jones, R. N. and Brown, S. D. M. 1991. Isolation of sequence common to A- and B- chromosomes of rye (Secale cereale) by microcloning. Plant Mol. Biol. Rep. 9: 21-30.

Scalenghe, F., Turco, E., Edstrom, J. E., Pirrotta, V. and Melli, M. I. 1981. Microdissection and cloning of DNA from a specific region of Drosophila melanogaster polytene chromosomes. Chromosoma 82: 205-216.

Schondelmaier, J., Martin, R., Jiahoor, A., Houben, A., Graner, A., Kopp, H. U., Herrmann, R. G. and Jung, C. 1993. Microdissection and microcloning of the barley (Hordeum vulgare L.) chromosome 1HS. Theor. Appl. Genet. 86: 629-636.

Seifertova, E., Zimmerman, L. B., Gilchist, M. J., Macha, J., Kubickova, S., Cernohorska, H., Zarsky, V., Owens, N. D., Sesay, A. K., Tlapakova, T. and Krylov, V. 2013. Efficient high-throughput sequencing of a laser microdissected chromosome arm. BMC Genomics 14: 357.

Shibata, F., Matsusaki, Y. and Hizume, M. 2017. Fluorescence in situ hybridization on chromosome spread of Pinus tabulaeformis. Cytologia 82: 99.

Taguchi, T., Kubota, S., Mezaki, T., Tagami, E., Sekida, S., Nakachi, S., Okuda, K. and Tominaga, A. 2016. Identification of homogeneously staining regions by G-banding and chromosome micro- 
dissection, and FISH marker selection using human Alu sequence primers in a scleractinian coral Coelastrea aspera Verrill, 1866 (Cnidaria). Comp. Cytogenet. 10: 61-75.

Tian, C., Lu, Y., Deng, J. and Liu, G. 1999. The cloning of HMWGS1Dx5 gene fragement using chromosome microdissection in wheat. Chin. J. Biotechnol. 15: 255-258.

Yanagi, T., Shirasawa, K., Terachi, M. and Isobe, S. 2017. Sequence analysis of cultivated strawberry (Fragaria $\times$ ananassa Duch.) using microdissected single somatic chromosomes. Plant Methods 13: 91.

Vega, M., Abbo, S., Feldman, M. and Levy, A. A. 1994. Chromosome painting in plants: In situ hybridization with a DNA probe from a specific microdissected chromosome arm of common wheat. Proc. Natl. Acad. Sci. U.S.A. 91: 12041-12045.

Wan, L., Wang, H., Zhou, Y., Bu, X., He, M. and Chen, Z. 2000. Microdissection and microcloning of the addition chromosome in wheat-wheatgrass alien addition line TAI-27 containing BYDV resistant gene. Chinese High Technol. Lett. 10: 10-14.
Yang, F., Trifonov, V., Ng, B. L., Kosyakova, N. and Carter, N. P. 2017. Generation of Paint Probes by Flow-Sorted and Microdissected Chromosomes. In: Liehr, T. (ed.). Fluorescence In Situ Hybridization (FISH). Springer Verlag, Berlin. pp. 412-456.

Zadesenets, K. S., Ershov, N. I. and Rubtsov, N. B. 2017. Wholegenome sequencing of eukaryotes: From sequencing of DNA fragments to a genome assembly. Russ. J. Genet. 53: 631-639.

Zhang, K., Martiny, A. C., Reppas, N. B., Barry, K. W., Malek, J., Chisholm, S. W. and Church, G. M. 2006. Sequencing genomes from single cells by polymerase cloning. Nat. Biotechnol. 24: 680-686.

Zhou, R. and $\mathrm{Hu}, \mathrm{Z}$. 2007. The development of chromosome microdissection and microcloning technique and its applications in genomic research. Curr. Genomics 8: 67-72.

Zlotina, A., Kulikova, T., Kosyakova, N., Liehr, T. and Krasikova, A. 2016. Microdissection of lampbrush chromosomes as an approach for generation of locus-specific FISH-probes and samples for high-throughput sequencing. BMC Genomics 17: 126. 